\title{
Colapso de traquéia em um cão
}

\author{
Tracheal collapse in a dog \\ Juliano dos Santos Evangelho ${ }^{1}$, Fabio dos Santos Teixeira ${ }^{2}$, Marianne Lamberts ${ }^{3}$ \\ $\&$ Ana Cristina Pacheco de Araujo ${ }^{4}$
}

\begin{abstract}
RESUMO
Este relato descreve a ocorrência de um caso de colapso traqueal cervical em um cão da raça Dachshund, fêmea, 2 anos de idade, atendida no Hospital de Clínicas Veterinárias da Universidade Federal do Rio Grande do Sul (HCV/UFRGS), que apresentava dispnéia não responsiva ao tratamento clínico. Foi tratado pelo método de correção cirúrgica através da utilização de próteses de seringas recortadas e dispostas em forma de "C", com o objetivo de circundar parcialmente a traquéia e evitar o seu achatamento dorsoventral. O cão após o $1^{\circ}$ dia de pós-operatório, apresentou dispnéia, na qual evidenciou-se o estreitamento da luz traqueal por edema. Após o $5^{\circ}$ dia da cirurgia o cão recebeu alta hospitalar, já não apresentando qualquer sinal de dispnéia. Não foi relatado pelo proprietário presença de tosse, nem observado durante o exame clínico, achado significativo citado pela literatura consultada. Este relato destaca a necessidade de uma avaliação clínica minuciosa do paciente, não baseando apenas nos sinais clínicos clássicos a esta doença. O diagnóstico final pode ser dificultado em virtude da gama de alterações anatômicas possíveis na traquéia.
\end{abstract}

Descritores: cão, traquéia, colapso de traqueal, próteses de seringa, tosse.

\begin{abstract}
This reports describes a tracheal collapse in a Dachshund, female, 2-year old dog, consulted at the Hospital de Clínicas Veterinárias da Universidade Federal do Rio Grande do Sul, Brazil (HCV-UFRGS), which presented dyspnea nonresponsive to clinical treatment. It was applied the technique of surgical correction through the use of syringe prosthesis disposed in a "C" shape with the purpose of partially embracing the trachea to avoid its dorsoventral flattening. On the first day after surgery the dog shown dyspnea due to the narrowing of the trachea light by edema, but after five days, no signal of dyspnea was observed. Cough was not observed by the owner at any moment, neither under pressure on the trachea at the clinical re-evaluation. This article points out the necessity of a careful clinical evaluation of the patient to obtain a definitive diagnostic of tracheal collapse, instead of only collecting the classical symptoms of this disorder. Sometimes the diagnostic can be dificult and to pass not perceived at clinical vets observation on rotinery attending, due to the several degress of traqueal anatomic alteration.
\end{abstract}

Key words: dog, trachea, tracheal collapse, syringe prosthesis, cough. 


\section{INTRODUÇÃO}

A traquéia é um órgão de tecido conjuntivo tubular flexível que se estende da cartilagem cricóidea da laringe até sua bifurcação dorsal na porção cranial da base do coração [3]. Há dois tipos de colapso de traquéia, as formas dorsoventral e laterolateral. $\mathrm{O}$ achatamento dorsoventral é comumente descrito e está associado a uma membrana dorsal pedunculosa [2]. A traquéia colapsada é encontrada freqüentemente com envolvimento cervical e torácico, podendo eventualmente envolver apenas a região cervical [9]. Ocorre tipicamente em cães de meia idade e idosos nas raças toy e miniatura $[5,6,11]$.

A primeira queixa é tosse improdutiva crônica descrita como "grasnar de ganso" e é mais intensa durante a excitação ou exercício. Os sinais podem exacerbarem-se caso uma doença subjacente se desenvolva, como bronquite infecciosa, alérgica ou insuficiência cardíaca [1,7]. Alguns autores, entretanto, relatam colapso traqueal em cães jovens e que não apresentam tosse, ou os que apresentaram, não observou da forma característica $[4,8]$.

O diagnóstico é baseado nos sinais clínicos e na radiografia da traquéia. As radiografias cervicais, obtidas na inspiração máxima, demonstrarão o colapso da traquéia extratorácica e as torácicas, obtidas na expiração máxima, mostrarão o colapso da traquéia intratorácica [10].

O tratamento cirúrgico deve ser considerado para animais que não respondem ao tratamento clínico e apresentam dispnéia importante. Dentre as técnicas sugeridas, destaca-se a da utilização de próteses de polipropileno ou com seringas plásticas recortadas, dispostas em forma de "C", com o objetivo de circundar parcialmente a traquéia e manter o formato anatômico original [7].

\section{CASO CLÍNICO}

Um cão da raça Dachshund, fêmea, 2 anos de idade, foi atendido no Hospital de Clínicas Veterinárias da UFRGS com histórico de dificuldade respiratória, emaciação progressiva, poliúria, polidipsia , disfagia e tratamentos anteriores sem resultado. O exame clínico revelou normotermia, mucosas hipocoradas, frequiência cardíaca de 120 batimentos por minuto, estertores úmidos bem audíveis a nível de traquéia cervical, dispnéia inspiratória sob agitação, sialorréia e aumento de linfonodo submandibular esquerdo.

A avaliação radiológica inicial do tórax e região cervical foi inconclusiva. No hemograma evidenciou-se discreta anemia com hematócrito $30 \%$ e leucometria com parâmetros normais. $\mathrm{O}$ animal foi submetido à anestesia para avaliação da cavidade oral, laringe e traquéia (Figura1). Foi observado corpo estranho linear aderido ao frênulo da língua, na palpação da traquéia evidenciou-se um achatamento dorsal da traquéia cervical. Em nova avaliação radiográfica da traquéia, realizada em inspiração máxima, foi evidenciado colapso da traquéia cervical dorsoventral (Figura 2).

\section{DISCUSSÃO E CONCLUSÕES}

O animal foi submetido à correção cirúrgica, com a utilização de próteses de seringas recortadas e dispostas em forma de "C" com o objetivo de circundar parcialmente a traquéia e evitar o achatamento dorsoventral da mesma,(Figuras 3a,3b,3c e 3d). A terapia antimicrobiana de amplo espectro e corticóide foi instituída com o objetivo de minimizar a possibilidade de infecção e edema pós-operatório.

O animal apresentou aumento de dispnéia inspiratória um dia após a cirurgia, sendo então submetido a nova avaliação radiográfica da traquéia cervical, quando foi constatado diminuição do lúmen traqueal por edema. Após o $5^{\circ}$ dia de pós-operatório o animal teve alta sem qualquer sinal clínico respiratório. O proprietário nunca observou tosse, que também não foi evidenciada à palpação no exame clínico, achado mais significativo citado pela literatura consultada.

O presente relato destaca a necessidade de uma avaliação minuciosa, embasados não apenas nos sinais clássicos a doença. Apesar do sinal mais comum da doença ser tosse crônica [1], alguns autores destacam a possibilidade dos cães não apresentarem este sinal clínico [4]. Dentre as técnicas cirúrgicas corretivas desta afecção, destacou-se a utilização de próteses de seringa em forma de "C" dispostas ao redor da traquéia, devido ao maior índice de sucesso com tal procedimento [7]. 


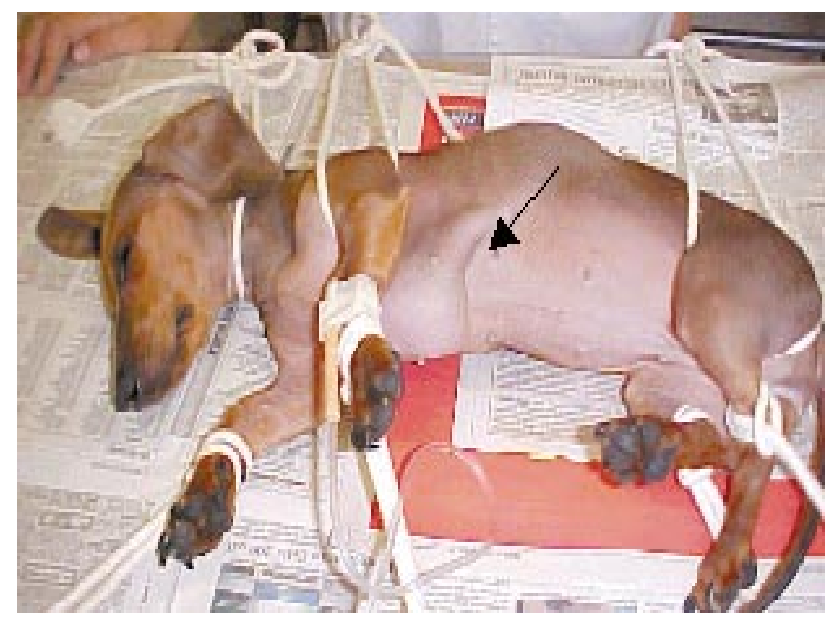

Figura 1. Cão com dificuldade respiratória, sendo evidenciado o esforço na inspiração (seta).

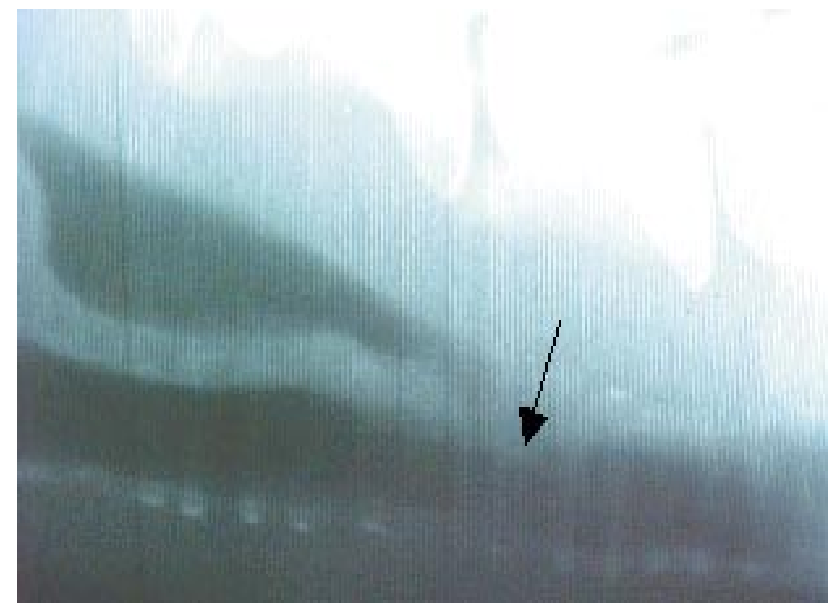

Figura 2. Estreitamento da luz traqueal (seta).

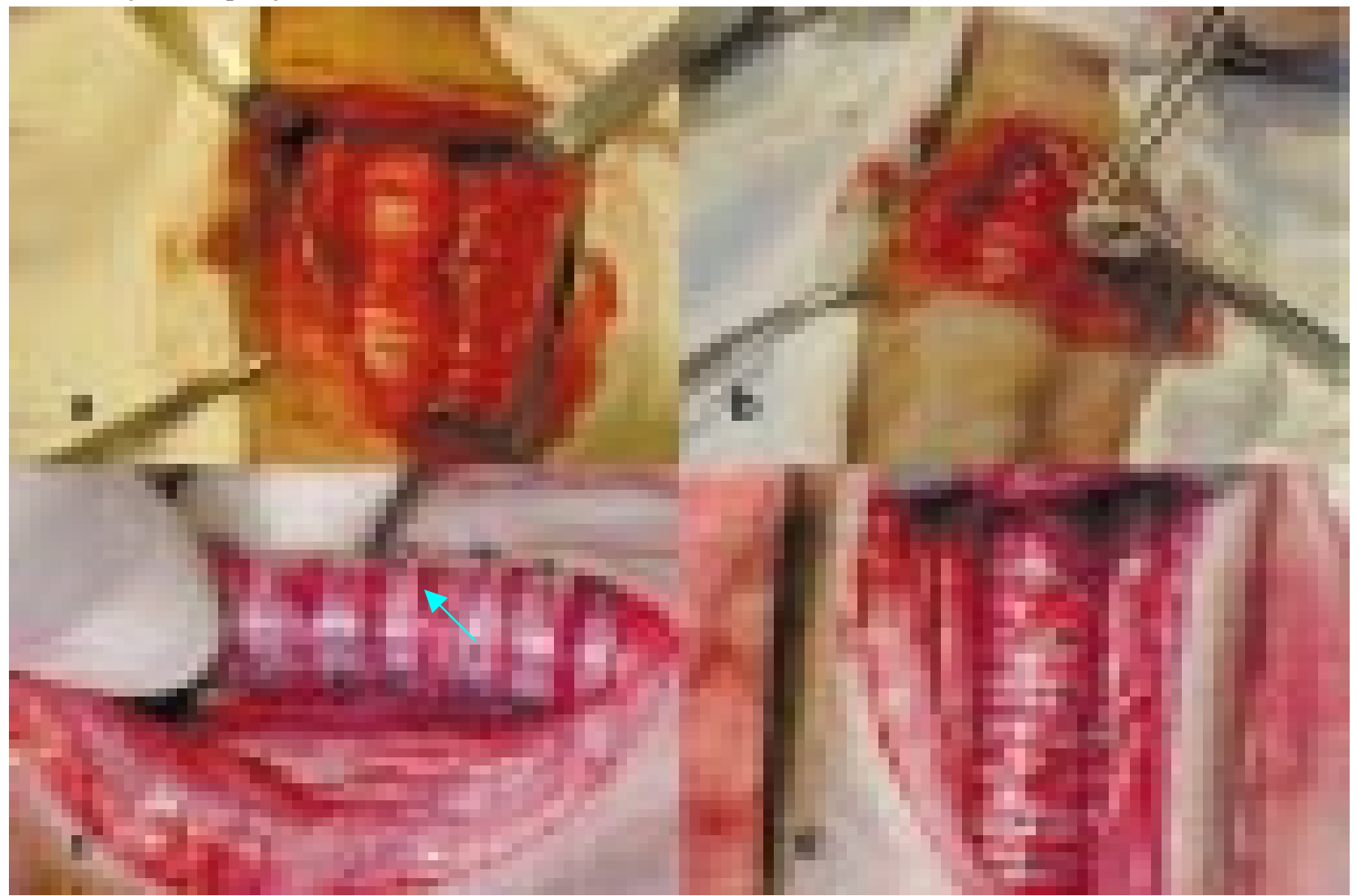

Figura 3. a) Local da traquéia colapsada (seta). b) Prótese de seringa. c) vista lateral da traquéia após correção cirúrgica. d) vista ventral da traquéia após correção cirúrgica.

\section{REFERÊNCIAS}

1 Burk R.L. \& Ackerman N. 1996. Small Animal Radiology and Ultrassonografy. Philadelphia: W.B.Saunders Co, pp. 101-105.

2 Ettinger S. \& Ticer J.W. 1992. Moléstias da Traquéia. In: Ettinger S.J. (Ed). Tratado de Medicina Interna Veterinária. v.2. São Paulo: Manole, pp.839-843.

3 Fingland R.B. 1996. Traquéia. In: Bojrab J.M. (Ed). Técnicas Atuais em Cirurgias de Pequenos Animais. 3.ed. São Paulo: Roca, pp.323-331

4 Fingland R.B. 1998. Diagnóstico e Tratamento Cirúrgico das Doenças Obstrutivas da Via Aérea Superior. In: Birchard S.J.; Sherding R.G. (Eds). Manual Saunders Clínica de Pequenos Animais.São Paulo: Roca, pp.617-630. 
5 Fossum T.W. 1997. Small Animal Surgery. St. Louis: Copyright, pp.632-637.

6 Harari J. 1996. The National Veterinary Medical Series Small Animal Surgery. Philadelphia: Willians \& Wilkirns, pp.7576.

7 Hawkins E.C. 2001. Distúrbios da traquéia e dos brônquios. In: Nelson, R.W.(Ed). Medicina Interna de Pequenos Animais. 2.ed. Rio de Janeiro: Guanabara Koogan, pp.228-229.

8 Hedlund C.S. 1991. Tracheal collapse. Problem Vetmed. 3: 229-238.

9 Schaer M., Ackerman N. \& King R. R. 1996. In: Ettinger S.J. (Ed). Manual de Medicina Interna Veterinária. São Paulo: Manole, pp. 331-332.

10 Shaw D. \& Ihle S. 1999. Medicina Interna de Pequenos Animais. 3.ed. Porto Alegre: Artes Médicas, pp. $236-237$.

11 Tillson D.M. 2003. “Tracheal Collapse - Are There Surgical Options?” Available from: URL: <http:// www.vetsoftware.com/NAVC_surgery12.htm>(20/05/2003). 